\title{
Complete Genome Sequence of Carbonic Anhydrase Producing Psychrobacter sp. SHUES1
}

\author{
Mengmeng $\mathrm{Li}^{1}$, Xuejiao Zhu ${ }^{1}$, Stephenson Wilkinson ${ }^{2}$, Minsheng Huang ${ }^{1}$ and \\ Varenyam Achal ${ }^{1 *}$ \\ ${ }^{1}$ Shanghai Key Lab for Urban Ecological Processes and Eco-Restoration, School of Ecological and Environmental Sciences, \\ East China Normal University, Shanghai, China, ${ }^{2}$ Department of Civil Engineering, University of Wolverhampton, \\ Wolverhampton, UK
}

Keywords: whole genome sequencing, Psychrobacter sp., carbonic anhydrases, urease, biocement

\section{OPEN ACCESS}

Edited by:

Prayad Pokethitiyook Mahidol University, Thailand

Reviewed by:

Christopher L. Hemme, University of Rhode Island, USA Mark Sutherland,

University of Bradford, UK

*Correspondence:

Varenyam Achal

varenyam@re.ecnu.edu.cn

Specialty section:

This article was submitted to

Microbiotechnology, Ecotoxicology

and Bioremediation,

a section of the journal

Frontiers in Microbiology

Received: 30 June 2016

Accepted: 30 August 2016

Published: 13 September 2016

Citation:

Li M, Zhu X, Wilkinson S, Huang M and Achal $V$ (2016) Complete Genome

Sequence of Carbonic Anhydrase

Producing Psychrobacter sp.

SHUES1. Front. Microbiol. 7:1442.

doi: 10.3389/fmicb.2016.01442
Recent advances in biotechnology have allowed the study of new bacterial strains, which can produce enzymes that can be used in the bioremediation of heavy metals. Microbially induced carbonate precipitation (MICP) is a recent well-recognized process that has the potential to precipitate heavy metals, mainly those with a valency of +2 (Kumari et al., 2016). There are two enzymes, urease, and carbonic anhydrase, that play an important role in the MICP process. The role of carbonic anydrase (EC 4.2.1.1) in MICP is generally underestimated and most of the studies in past mainly focus on urease-producing microorganisms (Li et al., 2013, 2014; Kumari et al., 2014).

In the present study, Psychrobacter sp. SHUES1 was isolated from frozen alkaline soil sample collected at Shanghai, China. This bacterium produced lipase and protease at $4^{\circ} \mathrm{C}$ in a plate assay. The ability of Psychrobacter sp. to show extracellular lipolytic activity at low temperatures is widely known (Xuezheng et al., 2010); however, the remarkable property of this strain was in the precipitation of heavy metals including cadmium and zinc in parallel to the MICP process. Therefore, to know the type of enzyme or genes involved in the process of metal precipitation, this research aims to sequence the whole genome of Psychrobacter sp. SHUES1, and thus provide a genomic insight into its behavior.

Genomic DNA from Psychrobacter sp. SHUES1 was extracted using the DNeasy Blood \& Tissue Kit (Qiagen, USA), and its quantity and quality were evaluated on the Qubit. The extracted DNA was subjected to whole-genome shotgun sequencing using the NEBNext Ultra DNA Library Prep Kit (Illumina, San Diego, CA). Library construction was performed with the following process: DNA fragmentation, end repair, adding " $A$ " to the $3^{\prime}$ end, adaptor ligation and amplification. After library construction, the generated cluster was sequenced on an Illumina HiSeq2500 sequencing system, according to a paired end $2 \times 125 \mathrm{nt}$ multiplex program. 13,716,515 raw reads resulted in 13,144,818 quality-filtered trimmed reads, yielding a not less than $3 \mathrm{Mb}$ genome size. De novo genome assembly was performed using SPAdes-3.5.0. After purification, the assembly produced $3,115,590 \mathrm{bp}$ of sequence across 115 contigs with an N50 of 47,049 bp, with a longest sequence of $182,144 \mathrm{bp}$, and a G+C content of $43.5 \%$ (Table 1). Gene prediction and annotation were carried out using Prodigal_v2.6.1, blastp in the National Center for Biotechnology Information (NCBI) "nr" database. Gene ontology (GO) functional annotation of genes was carried out using the blast2GO algorithm, dominated by the following features: biological process (44\%), molecular process (42\%), and cellular component (14\%). Clusters of Orthologous Groups (COG) annotation was carried out in the NCBI COG database using rpsblast. A total of 2627 protein-coding genes, 45 tRNA-coding genes, and 6 rRNA genes were predicted in the draft genome. 
TABLE 1 | Genome features of Psychrobacter sp. SHUES1.

\begin{tabular}{lc}
\hline Attributes & Value \\
\hline Genome size (bp) & $3,115,590$ \\
GC content (\%) & 43.57 \\
Protein coding genes & 2627 \\
tRNA genes & 45 \\
rRNA genes & 6 \\
ncRNA genes & 116 \\
GenBank accession no. & LXWA00000000
\end{tabular}

The most significant finding of the whole genome sequencing of Psychrobacter sp. SHUES1 was the presence of carbonic anhydrase gene in it. Carbonic anhydrase participates in all physiological processes dealing with $\mathrm{CO}_{2}$ and $\mathrm{HCO}_{3}$, such as cellular $\mathrm{pH}$ regulation, calcification, acid, and ion transport (Smith and Ferry, 2000; Achal and Pan, 2011). It catalyses the interconversion of $\mathrm{CO}_{2}$ and $\mathrm{HCO}_{3}$, which ultimately promotes the precipitation of calcium carbonate in the presence of $\mathrm{Ca}^{2+}$ ions. Although there are a number of genome sequences of Psychrobacter sp. deposited in NCBI database, this is the first characterization of the genome sequence of strain SHUES1, which produces carbonic anhydrase which has a significant role in metal bioremediation based on the ability to promote the precipitation of metal carbonates. This sequencing result also suggests the importance of carbonic anhydrase in the MICP process which is a novel element in this field of research.

The present study is especially valuable in the area of biomineralization based on MICP processes, in the bioremediation of metals and in the development of microbial concrete (biocement). Urease is the main enzyme responsible

\section{REFERENCES}

Achal, V., and Pan, X. (2011). Characterization of urease and carbonic anhydrase producing bacteria and their role in calcite precipitation. Curr. Microbiol. 62, 894-902. doi: 10.1007/s00284-010-9801-4

Kumari, D., Pan, X., Lee, D. J., and Achal, V. (2014). Immobilization of cadmium in soil by microbially induced carbonate precipitation with Exiguobacterium undae at low temperature. Int. Biodeter. Biodegr. 94, 98-102. doi: 10.1016/j.ibiod.2014.07.007

Kumari, D., Qian, X.-Y., Pan, X., Achal, V., Li, Q., and Gadd, G. M. (2016). Microbially-induced carbonate precipitation for immobilization of toxic metals. Adv. Appl. Microbiol. 94, 79-108. doi: 10.1016/bs.aambs.2015.12.002

Li, M., Cheng, X., and Guo, H. (2013). Heavy metal removal by biomineralization of urease producing bacteria isolated from soil. Int. Biodeter. Biodegrad. 76, 81-85. doi: 10.1016/j.ibiod.2012.06.016

Li, Q., Csetenyi, L., and Gadd, G. M. (2014). Biomineralization of metal carbonates by Neurospora crassa. Environ. Sci. Technol. 48, 14409-14424. doi: $10.1021 /$ es5042546 in such studies; however, in our study the urease gene was not present in Pyschrobacter sp. SHUES1. This indicates the importance of carbonic anhydrase, as a less studied secondary enzyme for the MICP process. It is hoped that this research will encourage other researchers to look for this carbonic anhydrase precipitation pathway when carrying out MICP studies.

\section{NUCLEOTIDE SEQUENCE ACCESSION AND CULTURE COLLECTION NUMBER}

The Psychrobacter sp. SHUES1 whole genome shotgun (WGS) project has been deposited at DDBJ/ENA/GenBank under the accession LXWA00000000. The version described in this paper is version LXWA01000000, and consists of sequences LXWA01000001-LXWA01000115. The detail information and data related to sequences LXWA01000001-LXWA01000115 can be accessed as well as downloaded at http://www.ncbi. nlm.nih.gov/Traces/wgs/wgsviewer.cgi?val=LXWA01\&search= LXWA01000000\&display=contigs. This strain has also been deposited in the China General Microbiological Culture Collection Center (CGMCC 1.15733).

\section{AUTHOR CONTRIBUTIONS}

ML and XZ, performed experiments; $\mathrm{MH}$ and VA, analyzed data; SW and VA, wrote manuscript.

\section{ACKNOWLEDGMENTS}

This work was supported by the National Natural Science Foundation of China under Grant number 41550110499.

Smith, K. S., and Ferry, J. G. (2000). Prokaryotic carbonic anhydrases FEMS Microbiol. Rev. 24, 335-366. doi: 10.1111/j.1574-6976.2000.tb 00546.x

Xuezheng, L., Shuoshuo, C., Guoying, X., Shuai, W., Ning, D., and Jihong, S. (2010). Cloning and heterologous expression of two cold-active lipases from the Antarctic bacterium Psychrobacter sp. G. Polar Res. 29, 421-429. doi: $10.1111 /$ j.1751-8369.2010.00189.x

Conflict of Interest Statement: The authors declare that the research was conducted in the absence of any commercial or financial relationships that could be construed as a potential conflict of interest.

Copyright (c) $2016 \mathrm{Li}$, Zhu, Wilkinson, Huang and Achal. This is an open-access article distributed under the terms of the Creative Commons Attribution License (CC $B Y)$. The use, distribution or reproduction in other forums is permitted, provided the original author(s) or licensor are credited and that the original publication in this journal is cited, in accordance with accepted academic practice. No use, distribution or reproduction is permitted which does not comply with these terms. 